\title{
Hybrid Processing of Stochastic and Subjective Uncertainty Data
}

\section{J. Arlin Cooper, Scott Ferson, Lev Ginzburg}

\section{Prepared by}

Sandia National Laboratories

Albuquerque, New Mexico 87185 and Livermore, California 94550

for the United States Department of Energy

under Contract DE-AC04-94AL85000

Approved for public releașe; distribution is unlimited.
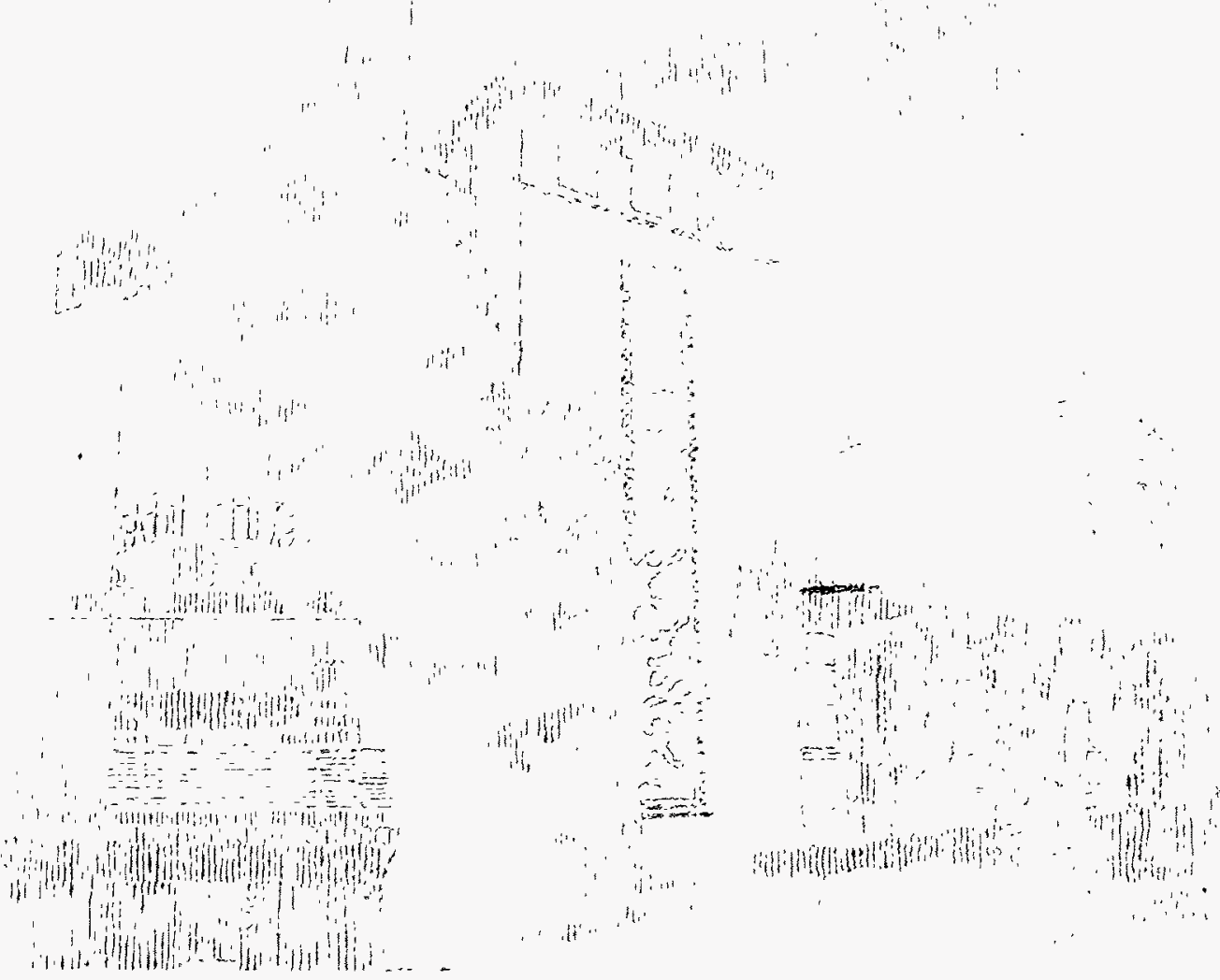
Issued by Sandia National Laboratories, operated for the United States Department of Energy by Sandia Corporation.

NOTICE: This report was prepared as an account of work sponsored by an agency of the United States Government. Neither the United States Government nor any agency thereof, nor any of their employees, nor any of their contractors, subcontractors, or their employees, makes any warranty, express or implied, or assumes any legal liability or responsibility for the accuracy, completeness, or usefulness of any information, apparatus, product, or process disclosed, or represents that its use would not infringe privately owned rights. Reference herein to any specific commercial product, process, or service by trade name, trademark, manufacturer, or otherwise, does not necessarily constitute or imply its endorsement, recommendation, or favoring by the United States Government, any agency thereof or any of their contractors or subcontractors. The views and opinions expressed herein do not necessarily state or reflect those of the United States Government, any agency thereof or any of their contractors.

Printed in the United States of America. This report has been reproduced directly from the best available copy.

Available to DOE and DOE contractors from

Office of Scientific and Technical Information

PO Box 62

Oak Ridge, TN 37831

Prices available from (615) 576-8401, FTS 626-8401

Available to the public from

National Technical Information Service

US Department of Commerce

5285 Port Royal Rd

Springfield, VA 22161

NTIS price codes

Printed copy: A03

Microfiche copy: $\mathrm{A01}$ 
SAND $95-2450$

Unlimited Release

Printed November 1995

Distribution

Category UC-706

\title{
Hybrid Processing of Stochastic and Subjective Uncertainty Data
}

\author{
J. Arlin Cooper \\ Sandia National Laboratories \\ Albuquerque, NM 87185-0490 \\ Scott Ferson \\ Applied Biomathematics \\ 100 North Country Road \\ Setauket, NY 11733 \\ Lev Ginzburg \\ State University of New York \\ Stony Brook, NY 11794
}

\begin{abstract}
$\underline{\text { Abstract }}$
Uncertainty analyses typically recognize separate stochastic and subjective sources of uncertainty, but do not systematically combine the two, although a large amount of data used in analyses is partly stochastic and partly subjective. We have developed methodology for mathematically combining stochastic and subjective data uncertainty, based on new "hybrid number" approaches. The methodology can be utilized in conjunction with various traditional techniques, such as PRA (probabilistic risk assessment) and risk analysis decision support. Hybrid numbers have been previously examined as a potential method to represent combinations of stochastic and subjective information, but mathematical processing has been impeded by the requirements inherent in the structure of the numbers, e.g., there was no known way to multiply hybrids. In this paper, we will demonstrate methods for calculating with hybrid numbers that avoid the difficulties. By formulating a hybrid number as a probability distribution that is only fuzzily known, or alternatively as a random distribution of fuzzy numbers, methods are demonstrated for the full suite of arithmetic operations, permitting complex mathematical calculations. It will be shown how information about relative subjectivity (the ratio of subjective to stochastic knowledge about a particular datum) can be incorporated. Techniques are also developed for conveying uncertainty information visually, so that the stochastic and subjective constituents of the uncertainty, as well as the ratio of knowledge about the two, are readily apparent. The techniques demonstrated have the capability to process uncertainty information for independent, uncorrelated data, and for some types of dependent and correlated data. Example applications are suggested, illustrative problems are worked, and graphical results are given.
\end{abstract}




$$
2
$$




\section{Table of Contents}

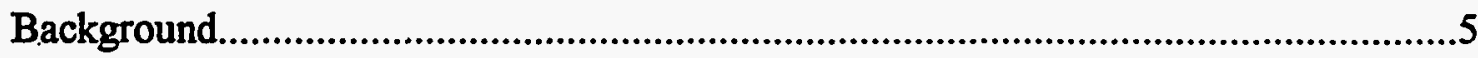

Subjective/Stochastic Hybrid Formulation...................................................................

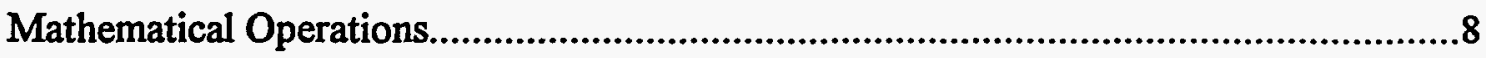

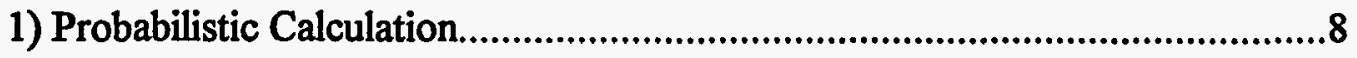

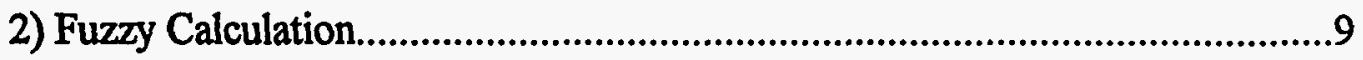

3) Separate Probabilistic/Fuzzy Calculation.....................................................10

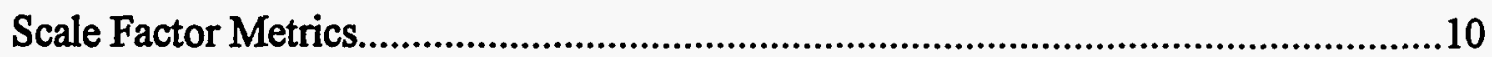

1) Uniform Scale Factors............................................................................

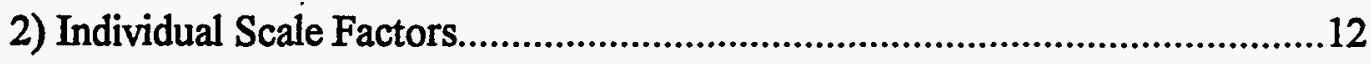

Software

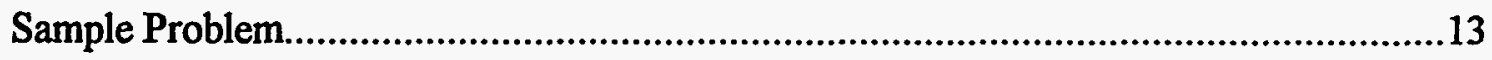

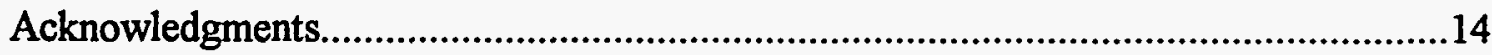

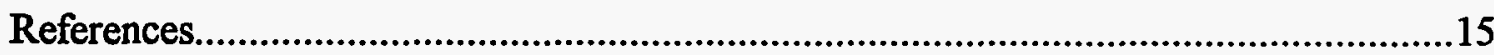

\section{Figures}

Fig. 1. Example Three-Dimensional Image opf a Hybrid Number......................................6

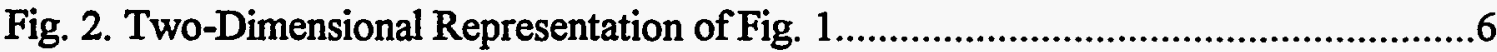

Fig. 3. Example of a Highly Stochastic Hybrid Number...............................................

Fig. 4. Example of a Highly Subjective Hybrid Number................................................

Fig. 5. Two Example Hybrid Operands for Multiplication.............................................9

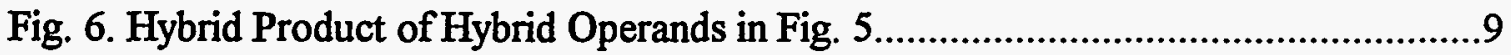

Fig. 7. A Fuzzy Number Randomly Selected from a Hybrid Number..............................10

Fig. 8. A Visual Depiction of a Scaled Hybrid Number.....................................................12 


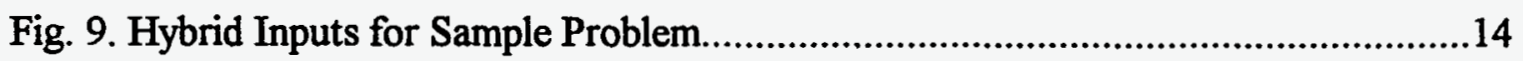

Fig. 10. Hybrid Output for Solution to Eqn. 7........................................................... 


\section{Background}

Subjective uncertainty can be propagated under a theory of fuzzy numbers with important differences from stochastic analysis [1]. These disparate forms of uncertainty are not readily combined in a single comprehensive theory. Kaufmann and Gupta [2] suggested that instead of confounding the two forms of uncertainty, they could be considered together but separately in a pair $(f, p)$, where $f$ is a fuzzy number and $p$ is a probability distribution. These pairs, which they termed "hybrid numbers," can be added together by convolving the respective elements according to normal rules for fuzzy arithmetic and

Portions of this work were supported by the United States Department of Energy under Contract DEAC04-94AL85000.

probability theory. Kaufmann [3] further explored the algebraic structure of such hybrid numbers. The Kaufmann/Gupta formulation of hybrid numbers allows addition and subtraction. Addition is defined as:

$$
\left(f_{1}, p_{1}\right)+\left(f_{2}, p_{2}\right)=\left(f_{1}+f_{2}, p_{1}+p_{2}\right)
$$

where the plus signs on the right side of the equation represent fuzzy max-min convolution $[1,2]$ and ordinary probabilistic sum-product convolution, respectively. This formulation of hybrid numbers does not directly allow multiplication or a full hybrid arithmetic (e.g., the product of a completely fuzzy number and a completely probabilistic number is undefined).

In this paper we describe other formulations for hybrid numbers that do permit multiplication, division, and other operations. These can be used in risk analysis problems where variability and subjectivity coexist. We propose that hybrid numbers can be thought of in two ways: a fuzzy probability distribution, or a random distribution of fuzzy numbers. These two concepts are equivalent, but have complementary interpretations and calculation strategies.

\section{Subjective/Stochastic Hybrid Formulation}

A hybrid number can be formulated as a probability that is only fuzzily known. Bounds on probability distributions can be used to express interval-type uncertainty about a random number in a pair of cumulative distributions that bound the random number's cumulative distribution. By nesting these probability distribution bounds at levels of presumption about the breadth of fuzzy uncertainty, we obtain a representation for hybrid numbers that is relatively simple to depict and understand.

A formal definition depends on monotonicity of the probability functions and nestedness of the fuzzy functions. Let $\mathbf{P}$ denote the set of cumulative probability functions defined on the real numbers $\mathbf{R}$, so each element $p \in P$ is a surjective function $p: R \rightarrow[0,1]$ such that 
$p\left(x_{1}\right) \leq p\left(x_{2}\right)$ whenever $x_{1}<x_{2}$. A hybrid number can be defined as a set of closed intervals, each characterized by a pair of functions from $P$ :

$$
\mathbf{H}:[0,1] \rightarrow \mathbf{P} \times \mathbf{P}: \alpha \mapsto\left(\mathrm{p}_{\alpha}, \mathrm{q}_{\alpha}\right)
$$

such that for $\alpha, \beta \in[0,1], p_{\alpha}(x) \geq p_{\beta}(x) \geq q_{\beta}(x) \geq q_{\alpha}(x)$ whenever $\alpha<\beta$. Here, $\alpha$ and $\beta$ represent fuzzy membership values ( $\alpha, \beta$ cuts) for values of $x$. The collection of cuts across $p(x)$ is from the set $F$ of fuzzy numbers. In Fig. 1, cumulative

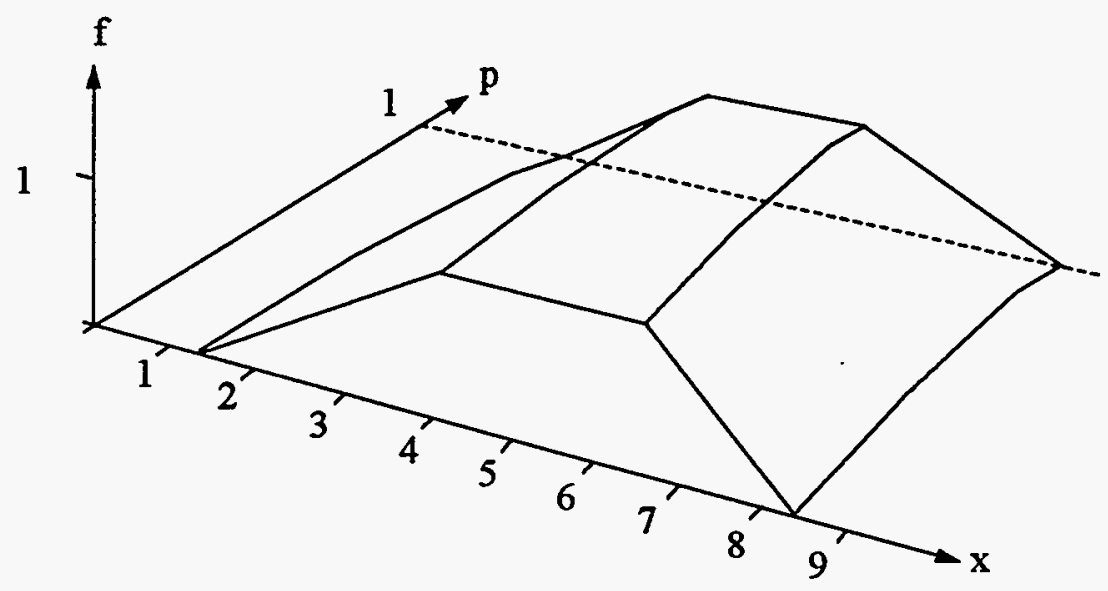

Figure. 1. Example Three-Dimensional Image of a Hybrid Number

probability functions $\mathrm{p}(\mathrm{x})$ and fuzzy uncertainty functions $\mathrm{f}(\mathrm{x})$ are shown in threedimensional form. When fuzzy functions can be represented by a few key vertices (e.g., three for triangular fuzzy numbers, four for trapezoidal fuzzy numbers), the twodimensional view of the three-dimensional form of Fig. 1 taken along the $f$ axis represents sufficient information without using the third dimension. This two-dimensional view is

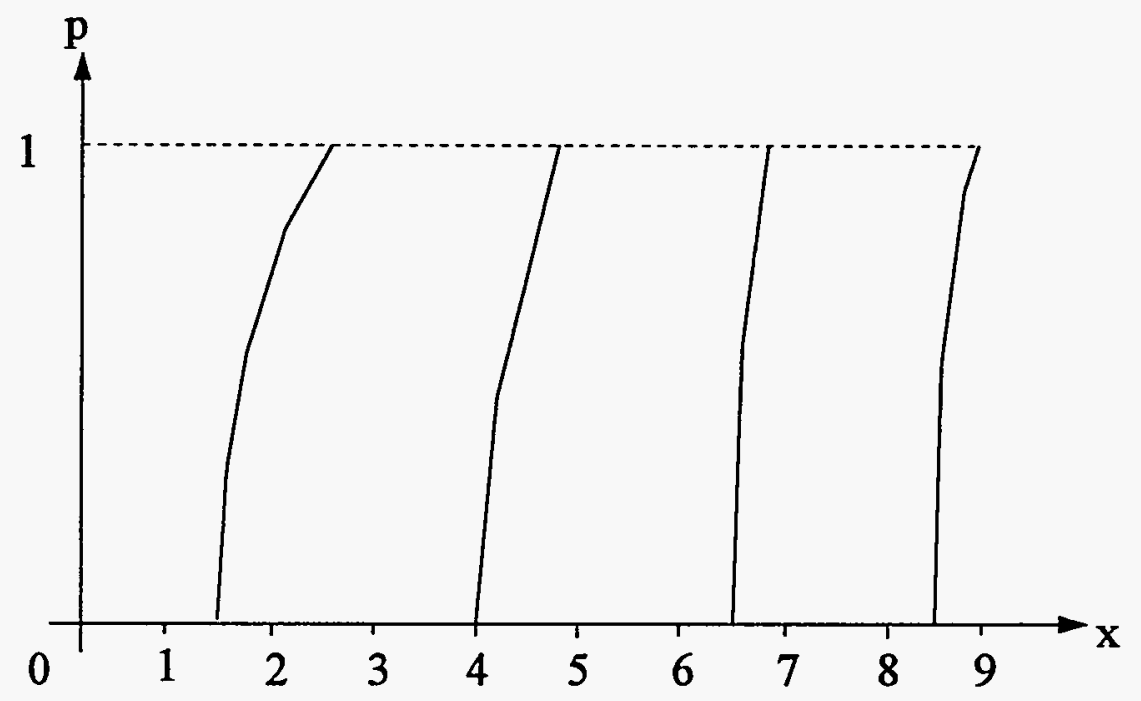

Figure 2. Two-Dimensional Representation of Fig. 1 
useful for computer routine output displays. The concept is illustrated in Fig. 2, where the four lines shown are loci for the four vertices of the trapezoidal fuzzy numbers in Fig. 1.

This formulation allows the full suite of arithmetic operations to be defined in ways to be described subsequently. The proposed hybrid numbers generalize probability distributions, fuzzy numbers, intervals, and scalars. When the image viewed along the $f$ axis shows $p$ functions that are nearly coincident, the hybrid number approaches a probability distribution that lacks fuzziness, as illustrated in Fig. 3.

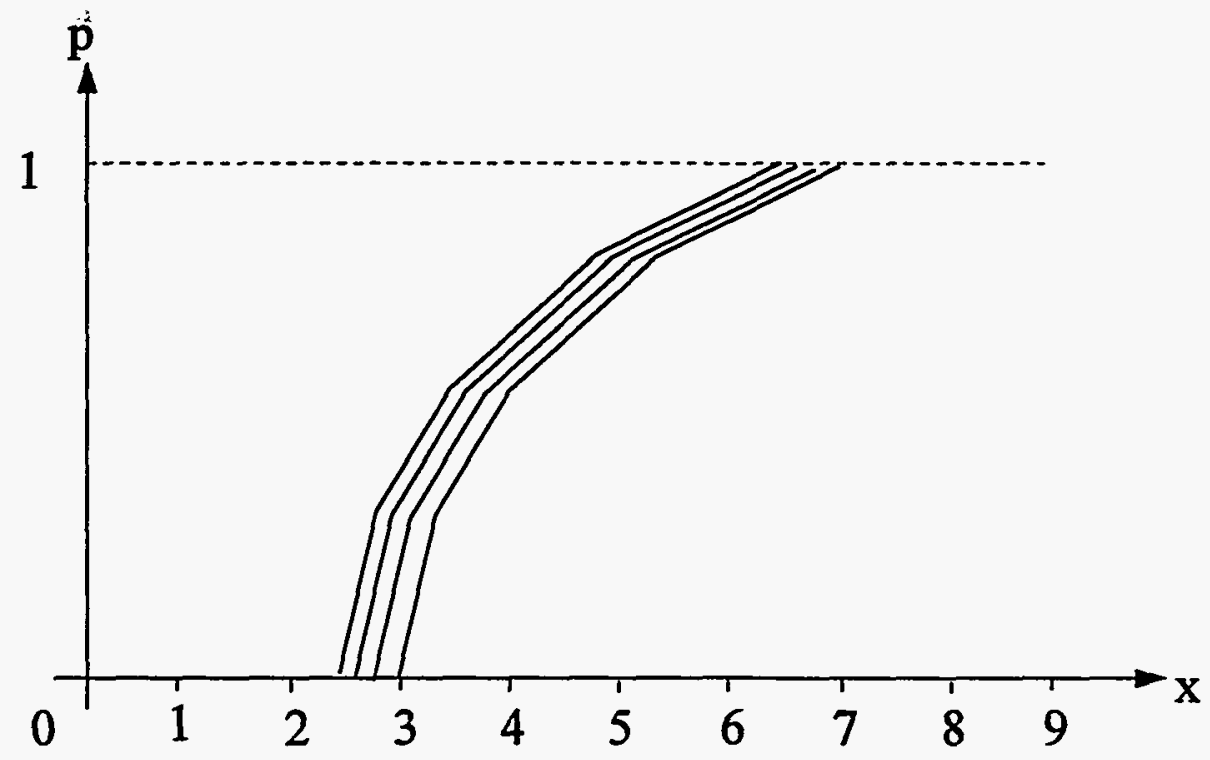

Figure 3. Example of a Highly Stochastic Hybrid Number

When the image viewed along the p-axis shows f functions that are nearly coincident as shown in Fig. 4 (or the view along the f-axis shows abrupt transitions of the $p$ functions

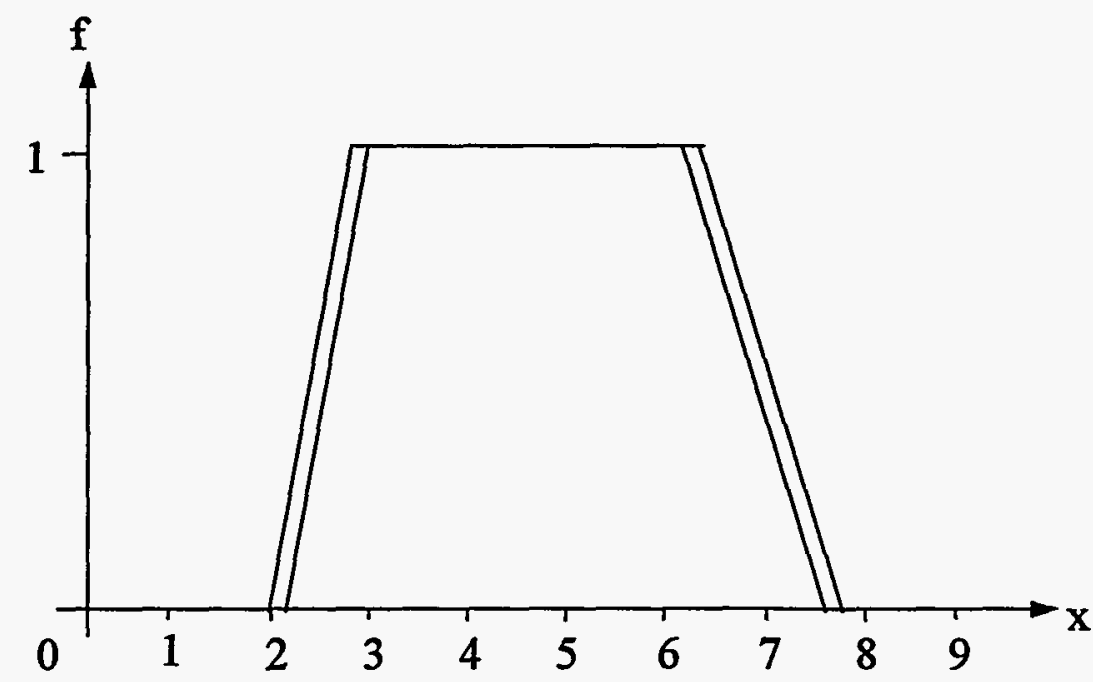

Figure 4. Example of a Highly Subjective Hybrid Number 
from zero to one), the hybrid number approaches a fuzzy number that lacks stochastic variability.

When the functions viewed along the $p$-axis have abrupt transitions between $f=0$ and $f=$ 1 , and the $p$ functions viewed along the $f$-axis have abrupt transitions between $p=0$ and $\mathrm{p}=1$, the hybrid number approaches an interval [4]. When the f functions viewed along the $\mathrm{p}$-axis and the $\mathrm{p}$ functions viewed along the $\mathrm{f}$-axis both show abrupt and nearly coincident transitions, the hybrid number approaches a scalar real number that lacks all uncertainty.

The formulation described in this section is mathematically equivalent to one based on fuzzy numbers and probability density functions. However, due to lack of monotonicity of density functions, the graphical images for hybrid numbers lack the appealing simplicity inherent in the cumulative distributions and will not be used here.

\section{Mathematical Operations}

1) Probabilistic Calculation. Algorithms for doing arithmetic with hybrid numbers can be built up from the lower-level algorithms that do arithmetic on pairs of bounding probability functions (Williamson and Downs [5]). Each of the arithmetic operations may be performed either with an assumption of independence, or without making any assumption about the stochastic dependence between the operands (in which case the bounds are generally wider). Applications of the methods to problems in risk analysis are illustrated in [6], [7]. A representative sample problem is illustrated in the final section of this paper.

Although the Williamson/Downs algorithms work very well for addition and subtraction, and can easily be extended for minimization and maximization, they only work for multiplication and division so long as the supports of the operands are nonnegative. Multiplication operands including negative regions (and division operand regions including zero so long as the divisor support does not include zero) can presumably be handled if additional algorithmic complexity is added.

An example will illustrate multiplication of two nonnegative hybrid numbers. Fig. 5 illustrates two hybrid operands for multiplication, where the fuzzy functions are trapezoidal.

The hybrid number on the left is centered near 2 and has values falling as low as 0.5 and rising as high as 3.5. The hybrid number on the right is centered near 6 and has values falling as low as 3.5 and rising as high as 9.5. The product of these two hybrid numbers 


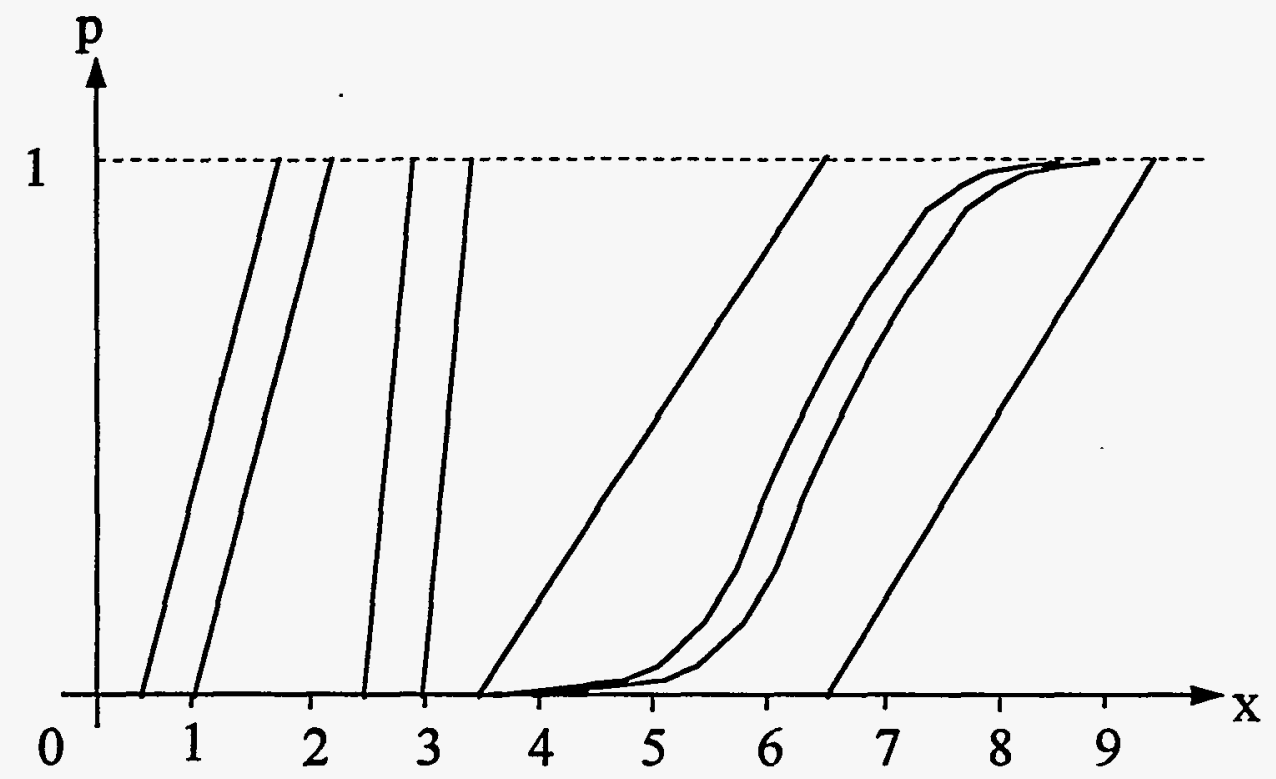

Figure 5. Two Example Hybrid Operands for Multiplication

(under an assumption of independence) is shown in Fig. 6. The product is centered near 15 and ranges almost from 2 to 30 .

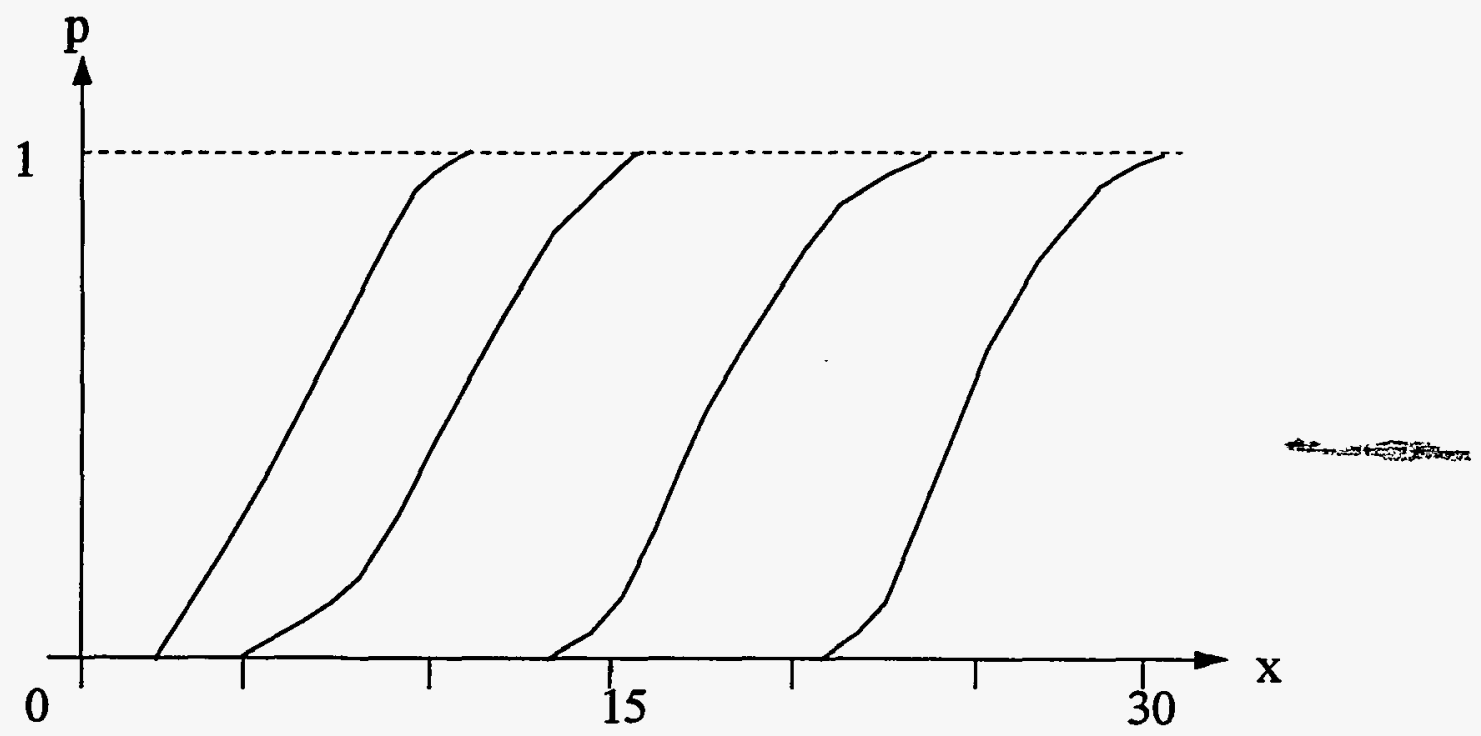

Figure 6. Hybrid Product of Hybrid Operands in Fig. 5

2) Fuzzy Calculation. One can also formulate hybrid numbers from a perspective in which they are probability distributions of elements that contain fuzziness. A formal definition of hybrid numbers that emphasizes this perspective is equivalent to the definition given above. We will describe one of the sampling strategies that can effect simulation solutions of the operations of hybrid arithmetic. Fuzzy number deviates from the hybrid number can be generated in the same way as for probability distributions of real numbers (Monte Carlo simulation or Latin Hypercube sampling). A random number is selected from the standard 
uniform distribution on $[0,1]$. It defines a horizontal cut through a three-dimensional hybrid operand at one value of $p$. An example of generating a random sample trapezoidal fuzzy number is illustrated in Fig. 7. The dashed line depicts a fuzzy cut corresponding to a sample of the $p$ functions at sample point $u$.

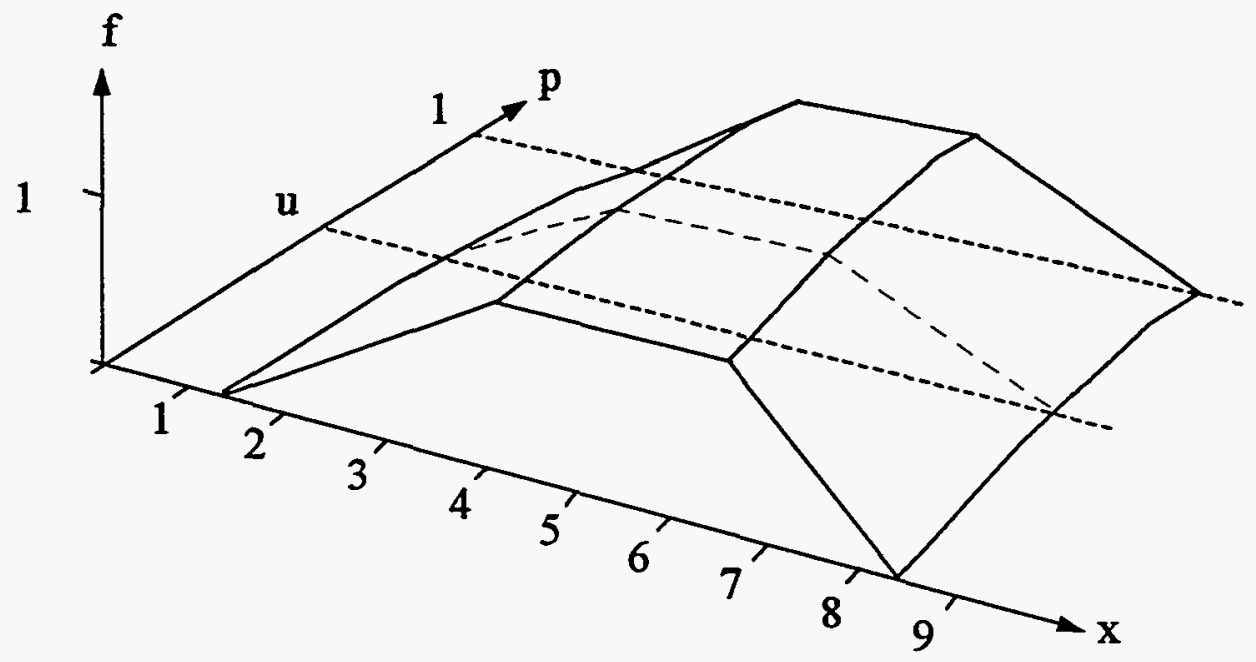

Figure 7. A Fuzzy Number Randomly Selected from a Hybrid Number

Another random number is selected to specify a fuzzy number cut through the other operand. The random fuzzy numbers generated are combined according to the rules of fuzzy arithmetic [2]. All of the standard arithmetic operations are defined, including addition, subtraction, multiplication, division, minimization, and maximization. Except for division by a fuzzy number whose support includes zero, these operations are closed in the set of fuzzy numbers.

3) Separate Probabilistic/Fuzzy Calculation. It can be appropriate (as illustrated in the following section) to compute probabilistic and fuzzy portions separately and to combine the two results in a hybrid representation. This is equivalent to combining stochastic information and fuzzy information separately, but portraying the results together for overall perspective. The use of hybrid formulations in this approach is mainly to associate the separate views in a more informative entity.

\section{Scale Factor Metrics}

When the values of input variables are not well known, risk analysts may expect to improve their analyses by incorporating new information that is learned through additional tests, accident assessments, etc. In a Bayesian sense, stochastic information can be improved. However, there are significant differences between Bayesian and fuzzy analyses. For example, a "non-informative" prior distribution in a Bayesian analysis might be considered an oxymoron by a fuzzy-algebra analyst, since even a uniform distribution is precisely defined. The general effect of Bayesian and other forms of probabilistic analysis is that extremes (tails of the distributions) are suppressed relative to the results of fuzzy analysis [8]. Since new input data may only slightly improve the stochastic knowledge 
about ill-defined situations such as abnormal environment responses, a non-Bayesian hybrid analysis has a useful role. A reasonable approach to this problem that does not assume nonexistent stochastic information is to provide for smooth transitioning from subjective (fuzzy) characterization to stochastic characterization as information about inputs is obtained.

1) Uniform Scale Factors. First, consider the case where the extent of knowledge about a problem is fractionally partitioned between stochastic and subjective portions. An input variable to an analysis whose variation characteristics are known partly stochastically and partly subjectively can be represented by a hybrid number with the relative stochastic/subjective information apportioned according to a scaling fraction:

$$
h(x)=\operatorname{axp}(x)+(1-a) \times f(x)
$$

where $a$ is an estimated scale factor representing the fractional stochasticity of the overall knowledge $(0 \leq a \leq 1)$, and where $x$ and + are operators on $x$ values.

The scale factor is a scalar, which Eqn. 3 suggests can be used to fractionally compress the abscissa (numeric) representation of the probabilistic constituent of variability by a, along with compression of the fuzzy constituent of uncertainty by (1-a). The total variation is then additive along the abscissa, i.e., a scaled sum of the two constituents.

A visual description of the uncertainty represented by a scaled hybrid number is shown in Fig. 8, for an example scale factor of $1 / 2$. The axes represent the numeric variability due to the constituents of stochastic knowledge and subjective knowledge. The dashed indication of a fuzzy function has been scaled down by a factor of two along the $\mathrm{x}$ axis from the subjective estimate. The dot-dashed indication of a probability function has also been scaled down by a factor of two from the stochastic estimate. The $x$-axis sum of the scaled variabilities is shown plotted as a three-dimensional hybrid number (solid lines).

This formulation is understood most clearly if the spread and shape of the fuzzy function and the probability function do not interact with each other, and if separate stochastic and fuzzy mathematics are used.

In the limited case for which the scale factor applies uniformly to all input variables in a mathematical analysis (and therefore also to the output), the conventional mathematical properties (identities, commutative property, and associative property for addition and multiplication, and multiplication distributive over addition and subtraction) hold for scalefactor arithmetic with no further requirements ${ }^{1}$.

\footnotetext{
${ }^{1}$ An important point is that variability for an operand must be entered only one time in equations in which there are multiple occurrences. For example, $A-A \equiv 0$. (We may be uncertain about the value of $A$, but not about the result of subtracting any value of $A$ from itself).
} 


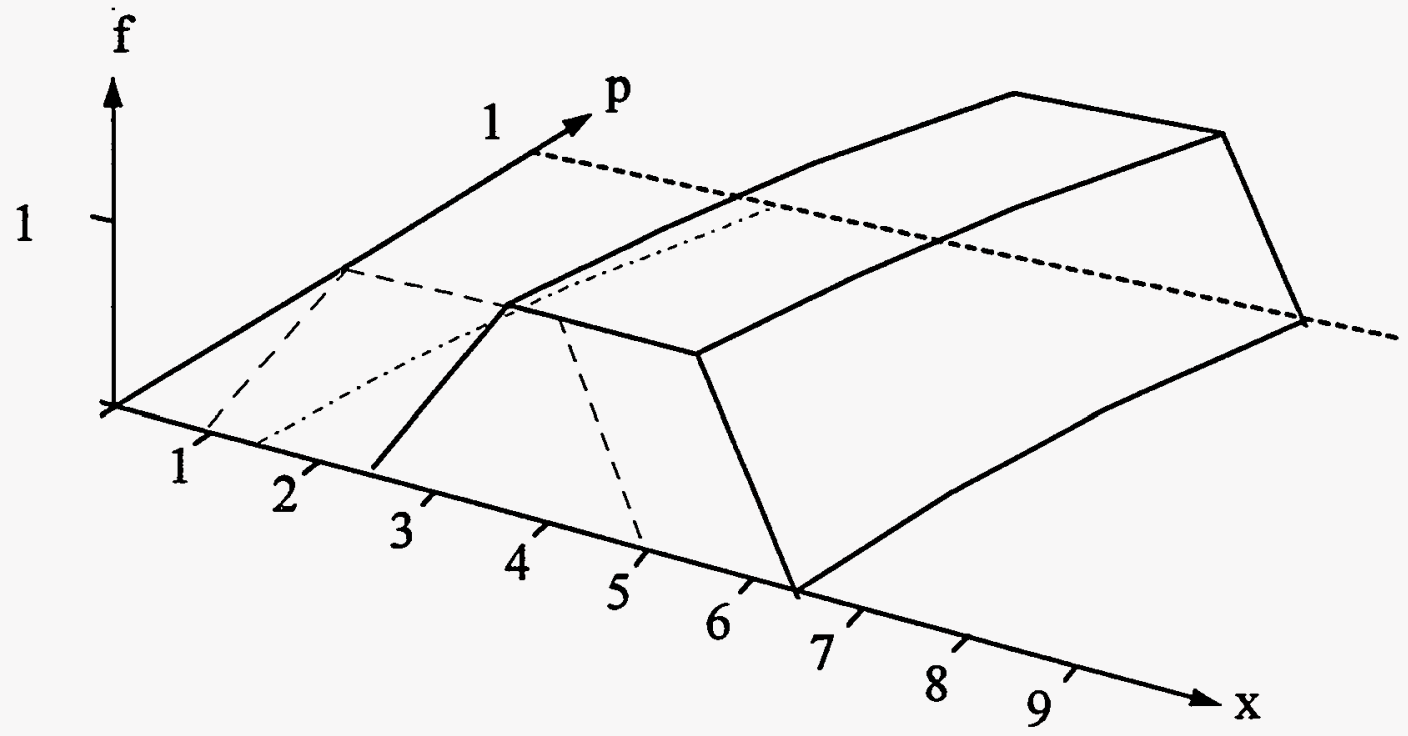

Figure 8. A Visual Depiction of a Scaled Hybrid Number

2) Individual Scale Factors. The more general case (allowing the scale factor to be individually chosen for each input operand), is of more practical interest. For this, a subscripted scale factor, $a_{i}$, will be introduced.

$$
h_{i}(x)=a_{i} \times p_{i}(x)+\left(1-a_{i}\right) \times f_{i}(x) .
$$

As one way to maintain the desired mathematical properties and to also assure that results meet physical expectations, we choose to average operand scale factors to obtain a resultant scale factor for multiplication and to use an average weighted by the relative contribution of the operands for addition. The following binary operations illustrate the concept:

$$
\begin{gathered}
h_{1}+h_{2}=\frac{a_{1} \hat{p}_{1}+a_{2} \hat{p}_{2}}{\hat{p}_{1}+\hat{p}_{2}}\left(p_{1}(x)+p_{2}(x)\right)+\frac{\left(1-a_{1}\right) \hat{f}_{1}+\left(1-a_{2}\right) \hat{f}_{2}}{\hat{f}_{1}+\hat{f}_{2}}\left(f_{1}(x)+f_{2}(x)\right) \\
h_{1} \times h_{2}=\frac{a_{1}+a_{2}}{2}\left(p_{1}(x) \times p_{2}(x)\right)+\frac{\left(1-a_{1}+1-a_{2}\right)}{2}\left(f_{1}(x) \times f_{2}(x)\right)
\end{gathered}
$$

where $\hat{p}_{i}$ and $\hat{f}_{i}$ represent either point estimates of $p_{i}(x)$ and $f_{i}(x)$, respectively, or functions (probabilistic and fuzzy, respectively); the operations on the $\mathrm{p}_{\mathrm{i}}(\mathrm{x})$ are ordinary sum-product convolution; and the operations on the $\mathrm{f}_{\mathrm{i}}(\mathrm{x})$ are fuzzy max-min convolution. The use of the logic represented by Eqns. 5 and 6 assures that the desired mathematical properties (e.g., associativity, commutativity, distributivity) will be maintained.

Eqns. 5 and 6 can be generalized to $n$ operands ( $n>2)$ by extending the number of subscripts in the equations from two to $\mathrm{n}$. Alternatively, $\mathrm{n}$ operands can be combined in successive binary operations by retaining information about prior operations. This is analogous to computing the average of the numbers $x, y$, and $z$ by averaging $x$ and $y$, 
multiplying the result by 2 (number of previous operands), adding $z$ and dividing by 3 (total number of operands).

Note that if the additive weighting ratios are consistent (e.g., $\frac{\hat{\mathrm{p}}_{1}}{\hat{\mathrm{p}}_{1}+\hat{\mathrm{p}}_{2}}=\frac{\hat{\mathrm{f}}_{1}}{\hat{\mathrm{f}}_{1}+\hat{\mathrm{f}}_{2}}$ ), the scale factors resulting from mathematical operations sum to one. This is an interesting but unnecessary property, e.g., a hybrid number could have a well known fuzzy constituent and a well known stochastic constituent.

The attribute of scale factors is that they provide a metric for the amount of relative knowledge about stochastic information and subjective information, supplementing the indications about the total amount of stochastic variability and subjective uncertainty shown qualitatively (e.g., in Figs. 3 and 4).

\section{Software}

One software package that is in use [8] permits addition, subtraction, multiplication, division, powers, exponentiation, logarithms, square roots, and a variety of other operations. Another [9] incorporates scale factors for each hybrid input variable and computes hybrid outputs along with scale factors for event tree and fault tree models. Both provide informative graphical representations.

\section{Sample Problem}

A sample problem (highly simplified version of an actual problem) is outlined here. An undesired event, $E$, can be the result of an incident, $A$, and an undesired response to the incident, $R$. The undesired response can be due to the failure of three subsystems, $I, T$, and $\mathbf{F}$, or due to a bypass of the three subsystems. Symbolically,

$$
E=A \wedge(R \mid A)=A \wedge(I \wedge T \wedge F \vee B)
$$

The hybrid input probabilities are shown in Fig. 9. 

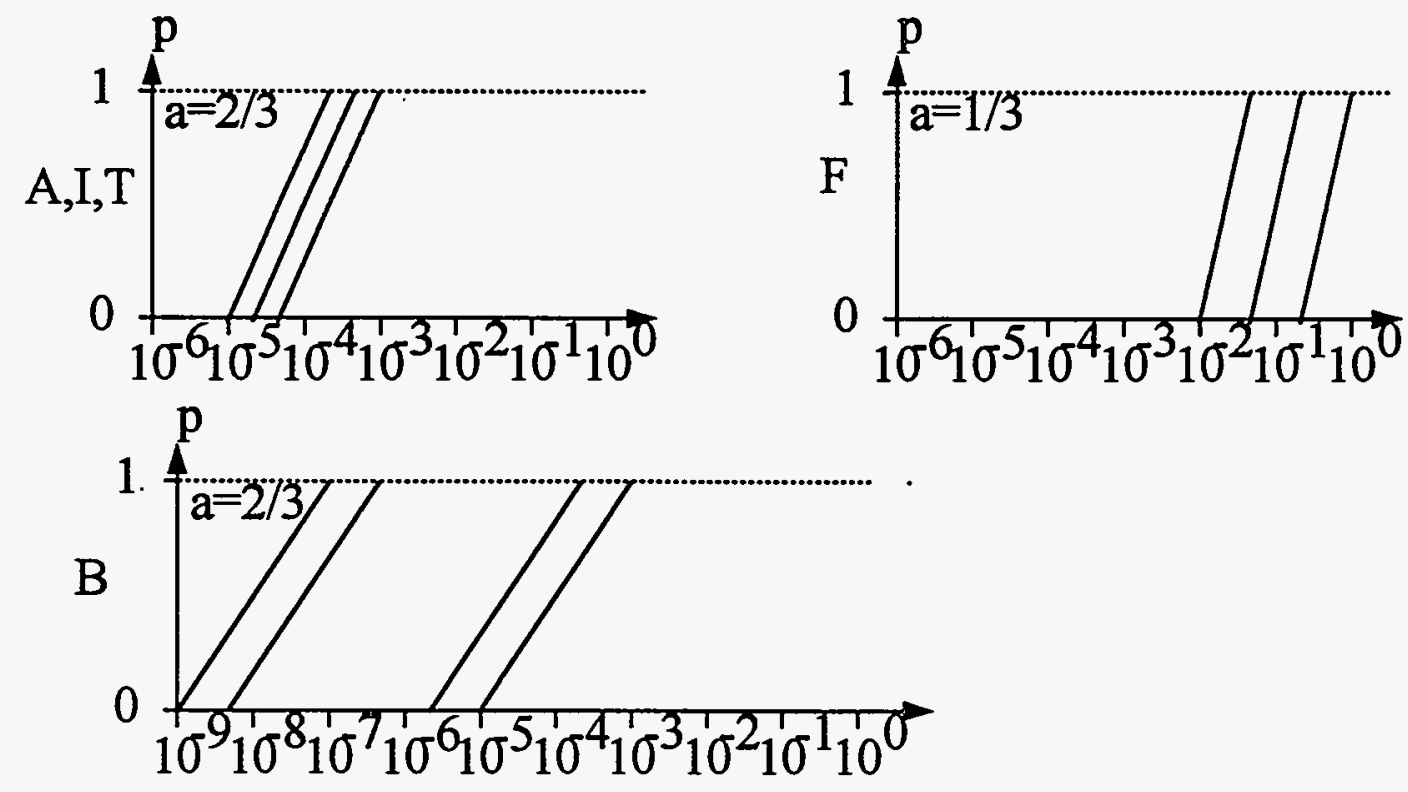

Figure 9. Hybrid Inputs for Sample Problem

The input probability axis is shown logarithmically. If failure of the three subsystems is independent, an arithmetic solution for $\mathrm{P}(\mathrm{E})$ is :

$$
P(E)=P(A) \times P(B)+P(A) \times[1-P(B)] \times P(C) .
$$

The Ref. 9 software was used to obtain the results shown in Fig. 10.

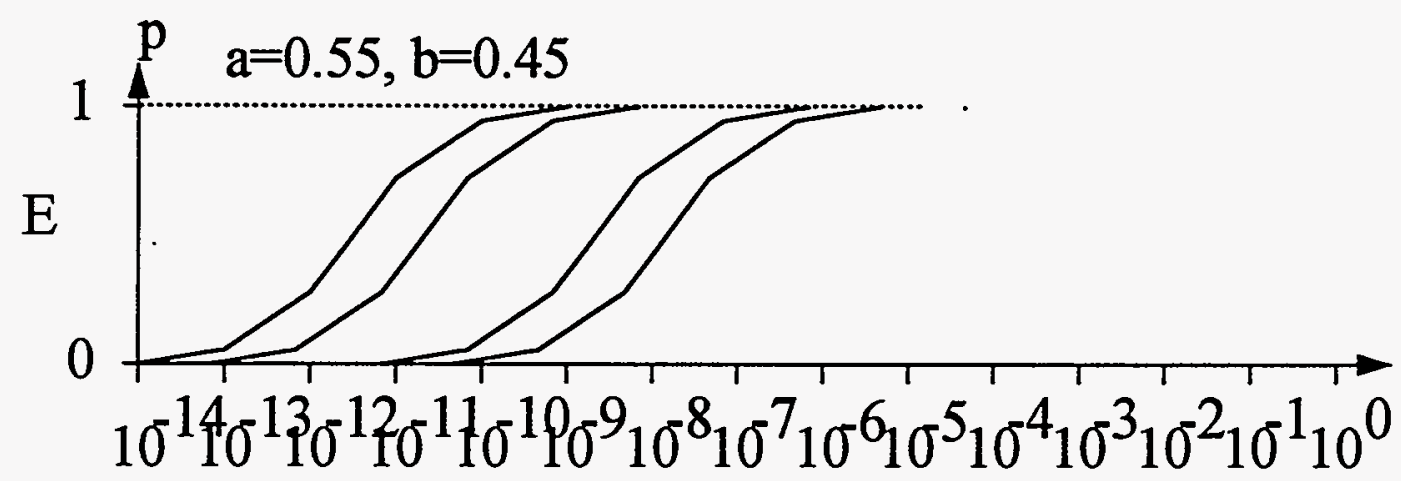

Figure 10. Hybrid Output for Solution to Eqn. 7.

\section{Acknowledgments}

Doug Cooper, Naval Research Laboratory, and Prof. George Klir, State University of New York at Binghamton, provided valuable assistance with the mathematical aspects of hybrid number processing. Rüdiger Kuhn, Bielefeld Universität, assisted with hybrid

\footnotetext{
${ }^{2}$ Although the exact arithmetic solution for probabilities of logically combined operands is straightforward for small problems, sophisticated software is required for most practical problems.
} 
mathematics and programming. Bob Roginski, Sandia National Laboratories created the hybrid scale factor software. 


\section{$\underline{\text { References }}$}

1. D. Dubois and H. Prade (1988) Possibility Theory: An Approach to Computerized Processing of Uncertainty, Plenum Press, New York.

2. A. Kaufmann and M. M. Gupta (1985) Introduction to Fuzzy Arithmetic: Theory and Applications, Van Nostrand Reinhold, New York.

3. A. Kaufmanin (1986) Hybrid data-- Various Associations between Fužzy Subsets and Random Variables, Fuzzy Sets Theory and Applications, NATO Advanced Science Series C: Mathematics and Physical Sciences 177, Reidel, Dordrecht and Boston.

4. A. Neumaier (1990) Interval Methods for Systems of Equations, Cambridge University Press, Cambridge.

5. R. C. Williamson and T. Downs (1990) Probabilistic Arithmetic I: Numerical Methods for Calculating Convolutions and Dependency Bounds, International Journal of Approximate Reasoning 4:89-158.

6. S. Ferson and T. F. Long (1994) Conservative Uncertainty Propagation in Environmental Risk Assessments, Environmental Toxicology and Risk Assessment- Third Volume, ASTM STP 1218, J. S. Hughes, G. R. Biddinger and E. Monos (eds.), American Society for Testing and Materials, Philadelphia.

7. S. Ferson, L. R. Ginzburg, and H. R. Akcakaya (1995), Whereof One Cannot Speak: When Input Distributions are Unknown, Risk Analysis [in press].

8. R. Kuhn and S. Ferson (1994) Risk Calc: Uncertainty Analysis with Interval and Fuzzy Arithmetic, Applied Biomathematics, Setauket, NY.

9. J. Arlin Cooper (1995) Fuzzy Algebra Uncertainty Analysis (NST 405) Workbook, Sandia National Laboratories. 
Distribution:

P. N. Demmie, 2415 MS 0303

C. F. Briner, 2674 MS 0328

M. J. DeSpain, 2674 MS 0328

D. M. Kunsman, 4111 MS 0415

A. C. Payne, Jr., 4115 MS 0425

W. R. Burcham, 5123 MS 0485

K. Ortiz, 5153 MS 0475

C. B. Richărdson, 5167 MS 0481

T. S. Edrington, 5205 MS 0459

M. S. Elpel, 5354 MS 9203

L. R. Gilliom, 5603, MS 0458

P. E. Rexroth, 5822 MS 0761

A. L. Camp, 6412 MS 0747

S. L. Daniel, 6412 MS 0747

M. J. Martinez, 6613 MS 0746

S. Neuhauser, 6641 MS 0718

R. H. Yoshimura, 6641 MS 0718

Technical Library, 4414 MS0899 (5)

Print Media, 12615 MS0619 $^{\circ}$

G. Pirtle, 7255 MS 1036

Document Processing for

DOE/OSTI, 7613-2 MS 0100 (2)

T. E. DeLano, 8114 MS 9201

D. S. Shạh, 8113 MS 9410

R. Zurn, 8114 MS 9201

C. W. Pretzel, 8414 MS 9408

Central Technical Files, 8523-2 MS

9018

Judy Lim, 8910 MS 9011

K. M. Hansen, 9133 MS 0844

W. C. Fan, 9352 MS 1166

S. K. Fletcher, 9411 MS 0777

M. Murphy, 9411 MS 0777

R. M. Jansma, 9415 MS 0777

W. C. Nickell, 12300 MS 0631

R. G. Easterling, 12302 MS 0829

R. E. Smith, 12302 MS 0491

K. V. Diegert, 12323 MS 0829

P. E. D'Antonio, 12324 MS 0491

J. P. Berry, 12324 MS 0491

M. Caldwell, 12324 MS 0491

J. M. Covan, 12324 MS 0491

L. W. Dahlke, 12324 MS 0491

M. E. Ekman, 12324 MS 0491

D. Isbell, 12324 MS 0491

J. Leyland, 12324 MS 0491

R. D. Pedersen, 12324 MS 0491

L. Vaughn, 12324 MS 0491

P. W. Werner, 12324 MS 0491

K. S. Ricker, 12326, MS 0638

S. D. Spray, 12331 MS 0490

R. R. Bennett, 12331 MS 0490

J. A. Cooper, 12331 MS 0490 (20)

J. P. Hoffman, Jr. , 12331 MS 0490

W. E. Mauldin, 12331 MS 0490

C. A. Trauth, Jr. , 12331 MS 0490

G. A. Sanders, 12332 MS 0492

J. P. Cates, 12332 MS 0492

P. J. Konnick, 12332 MS 0492

D. R. Lewis, 12332 MS 0492

D. H. Loescher, 12332 MS 0492

D. R. Olson, 12332 MS 0492

J. A. Richardson, 12332 MS 0492

C. G. Shirley, 12332 MS 0492

D. A. Summers, 12332 MS 0492

J. F. Wolcott, 12332 MS 0492

D. D. Carlson, 12333 MS 0405

D. E. Bennett, 12333 MS 0405 
R. J. Breeding, 12333 MS 0405

M. A. Dvorack, 12333 MS 0405

M. K. Fuentes, 12333 MS 0405

N. R. Grandjean, 12333 MS 0405

J. V. Hancock, 12333 MS 0405

T. R. Jones, 12333 MS 0405

S. A. Kalemba, 12333 MS 0405

Y. T. Lin, 12333 MS 0405

W. McCulloch, 12333 MS 0405

R. J. Roginski, 12333 MS 0405

K. B. Sobolik, 12333 MS 0405

CDR Tom Walker

Field Command, Defense Nuclear

Agency

FCFA

Kirtland AFB NM 87115-5000

Capt. James Schoeneman

Det. 1, AFSA/SENA

Kirtland AFB NM 87117-5000

John Lederer

OL-NS/EN

1651 First St. SE

Kirtland AFB NM 87117-5617

Harvey Dayhoff

OL-NS/ENBS

1651 First St. SE

Kirtland AFB NM 87117-5617

Al Matteucci

OL-NS/ENX1

1651 First St. SE

Kirtland AFB NM 87117-5617
J. L. Tenney, 12333 MS 0491

R. O. Winchester, 12333 MS 0405

G. C. Novotny, 12334 MS 0627

S. Humbert, 12334 MS 0627

E. L. Fronczak, 12334 MS 0627

J. E. Stayton, 12334 MS 0627

F. G. Trussell, 12334 MS 0627

J. M. Sjulin, 12335 MS 0830

D. S. Hill, 12367 MS 0636

P. D. Sands, 13221 MS 0811

Mark LeDoux

OL-NS/ENN

1651 First St. SE

Kirtland AFB NM 87117-5617

Prof. Nancy Leveson

Computer Science Engineering

Department

University of Washington

Seattle WA 98195

Dr. Scott Ferson

Applied Biomathematics

100 North Country Road

Setauket, NY 11733

Prof. Lev Ginzburg

Ecology and Evolution

State University of New York

Stony Brook, NY 11794 